\title{
A Mobile Learning Framework in the Cloud
}

\author{
Shanshan CHENG, Zenggang XIONG, Xueming ZHANG \\ School of Computer and Information Science ,Hubei Engineering University, China
}

\begin{abstract}
Mobile cloud learning, a combination of mobile learning and cloud computing, is a relatively new concept that holds considerable promise for future development and delivery in the education sectors. Cloud computing helps mobile learning overcome obstacles related to mobile computing. This paper proposes an interdisciplinary approach for design and development of mobile applications in the cloud. The approach includes front service toolkit and backend service toolkit. The front service toolkit packages data and sends them to a backend deployed in a cloud computing platform. The backend service toolkit manages rules and workflow of web services, supports fault tolerance and then transmits required results to the front service toolkit.
\end{abstract}

KEYWORD: Cloud Computing, Mobile Learning System, Mobile Devices

\section{INTRODUCTION}

Together with the explosive and rapid growth of Internet, mobile networks, mobile applications, and cloud computing, mobile cloud computing is introduced as a potential technology for mobile devices. As mobile network infrastructures continuously improve, their data transmission becomes increasingly available and affordable, and thus they are becoming popular clients to consume any internet web based applications. Cloud computing provides delivery of services, software and processing capacity over internet, reducing cost, increasing, automating systems, decoupling of service delivery from underlying technology, and providing flexibility and mobility of information. Mobile Cloud Computing (MCC) integrates the cloud computing into the mobile environment and overcomes the obstacles related to the performance (battery life, storage, and bandwidth), environment (heterogeneity, scalability and availability), and security (reliability and privacy). One future potential application of MCC is the Mobile Distance Learning (MDL), where the students can get the knowledge from centralized shared resources at any place and any time.

\section{CONCEPTS AND PRINCIPLES}

\subsection{Mobile Cloud Computing}

Mobile Cloud Computing at its simplest refers to an infrastructure where both the data storage and the data processing take place outside of the mobile device. Mobile cloud applications move the computing power and data storage away from mobile phones and into the cloud, bringing applications and mobile computing to not just smart phone users but a much broader range of mobile subscribers.

The mobile devices are connected to the mobile networks through base stations that establish and control the connections (air interface) and functional interfaces between the networks and mobile devices. Mobile users'request and information are transmitted to the central processors that are connected to the servers providing mobile network services. Here, services like AAA (Authentication, Authorization and Accounting) can be provided to the users based on Home Agent (HA) and subscribers'data stored in databases. The subscribers' requests are then delivered to a cloud through the Internet. Cloud controllers present in the Cloud, process the requests to provide the mobile users with the corresponding cloud services. These services are developed based on the concepts of utility computing, virtualization and service-oriented architecture.

\subsection{Mobile Cloud Computing learning}

The "mobile" in "mobile learning" has two implications: 
- Learner mobility: learners are able to engage in educational activities without the constraints of having to do so in a tightly delimited physical location. To a certain extent, learning can happen outside a classroom or in various locations, requiring nothing more than the motivation to do so wherever the opportunity arises from books, electronic resources, places and people.

- Mobile devices: portable, lightweight devices that are sometimes small enough to fit in a pocket or in the palm of one's hand. Typical examples are mobile phones, smart phones (like the iPhone), palmtops, and handheld computers like the iPad or PDAs (Personal Digital Assistants); Tablet PCs, laptop computers and personal media players like the iPod can also fall within its scope. These devices can be carried around with relative ease and used for communication and collaboration, and for teaching and learning activities that are different from what is possible with other media.

\subsection{Benefits of mobile cloud learning}

Traditional mobile learning must deal with the following drawbacks-high device and network costs, low network transmission rates, and limited education resources available. Combining the advantages of mobile learning and cloud computing, mobile cloud learning is introduced to solve these limitations (Kitanov \& Davcev, 2012).

Weber (2011) argues "greater connectivity between centralized server-side applications and low cost/low processor capacity mobile devices could provide better access, more control, and greater freedom for e-learners"

Mobile cloud learning has a number of benefits to both the content providers and the learners. First and foremost, it costs less. For content providers, it saves the large initial cost, spending on hardware and software incurred when installing all kinds of systems (Freeman, 2000). By paying regular monthly fees, even small schools and universities, which cannot justify the return-on-investment for the high initial setup costs can provide mobile cloud learning services without spending large set-up costs for infrastructure (Hirsch \& $\mathrm{Ng}, 2011$ ). In addition, they do not need to instantly maintain and upgrade hardware or software. Meanwhile, because all computing, storage, and updates are completed on the cloud side, mobile devices serve for display only (Mohamudally, 2011). As a result, users can utilize web-based applications on their mobile devices with small memory spaces because there is no need for software loading and document saving (Rao, Sasidhar, \& Kumar, 2010). They can use variable mobile devices to access learning content without purchasing, installing, or updating any software.

\subsection{Characteristics of mobile cloud learning}

Mobile cloud learning has the following characteristics.

-Storage and sharing: Learning outcomes and resources can be stored in the "Cloud," which provides almost unlimited store and computation capacities. Documents can be commonly edited and shared in the "Cloud," such as services provided by GoogleDocs, Live Skydrive, and Office Live.

- Universal accessibility: Learners can study as long as they have access to the network. Mobile cloud learning also makes a low-cost access terminal possible, because software, applications, and data are all operated in the cloud servers. This improved accessibility can greatly benefit developing regions.

- Collaborative interactions: Learners can cooperate anywhere in the "Cloud." From social learning perspectives, they can collaboratively build common knowledge through frequent and convenient interactions.

- Learner cantered: Mobile cloud learning is heavily people-oriented, which meets the individual needs of learners. Learners in the "Cloud" select suitable resources and can track their learning progress and outcomes.

\section{FRAMEWORKS}

In this section, the frameworks of the front service toolkit and backend service toolkit are proposed. The front service toolkit and backend service toolkit can work individually and only performs the cooperation with each other when the user requirements. Cloud computing, as a new concept for hosting and managing different services, is an environment solution for big data. It can eliminate the requirements of provisioning of users and allows owners of both small and enterprises to increase resources only when there is a rise in service demand.

A. The Framework of Front Service Toolkit Packager: All of the packagers are utilised for packaging information and data from users and then the packaged messages are transmitted to backend services in the cloud. In particular, the XML packager has the responsibility for organizing data according to XML schema defined in advance.

Analyser: Analysers consists of variable analyser, array analyser and XML analyser. They are corresponding to the above packagers separately, which are applied to extract information and data from packages routed from the backend service toolkit. As the interface of receiving messages, they are required to be identical to the packagers.

Applications need to compress and decompress the messages from users with various formats to the backend side if they require communication with database to record users' information, business flow 
and logging messages. Therefore, the compressing and decompressing functions are packaged as packager and analyser modules.

B. The Framework of the Backend Service Toolkit The backend service toolkit is the connection between the front side of applications and database. It is responsible for convert raw messages from clients into specified data format designed by developers and database designers. In this section, the framework of the backend service toolkit is proposed. The framework is mainly composed of analyser and packager, business services pool, and Infrastructure as a Service (IaaS). Note that the designed framework in this paper includes business process services pool possessing rule controlling services and process controlling services. Rules can be specified and designed by developers.Based on the application business detailed services, process controlling services control and manage the overall the business flow and message flow of mobile applications.

Analyser and packager: Analysers and packagers of the backend service toolkit have the same main functions as the front service toolkit. The front service toolkit uses these functions to trigger the transmission of data and messages to backend.

Application business detailed service pool: It is used for containing and deploying components and services of applications. Note that mobile applications are divided into functions as web services in the cloud. The cloud computing platform publishes web services through a Web server. Web services implementing application business have hierarchical relationships. Web services in this layer can invoke each other and, due to specific user requirements, can work together to complete application functions.

Business process services pool: This layer is a web service container which is integrated with the rule engine and workflow engine. They offer unique standard web service interfaces to external systems and mobile devices. Workflow engine organise the relationships among web services and defines main work flow and message flow. Therefore, the rule engine can deal with decisions of work flow in terms of application requirements. In particular, when there is more than one work flow in applications, the rule engine is considered to handle the flow of information and messages.

Database management services pool: This applies mapping techniques to map objects from the above layers into records in database. Here, two categories, namely, relationship database and XML database, are utilised. The backend program makes use of database management ervice sets to store data into databases and to retrieve record from tables. The backend service toolkit employs multiply databases to store data so as to restore storage if the main database crashes.
Fault detection services: This has the responsibility for detection and fault tolerance of faults of the front and backend service toolkits. The backend service toolkit includes service backup functions, which can back upcentral services and failure-prone services.

Generic service set: Common and generic services are abstracted and placed in this layer. This layer provides services to developers and other service sets. Developers can exploit libraries of services in this set to develop other applications.

Security services and QoS optimisation services: Developers and end users can define and put their security services into this module. The security module and QoS optimsation module work together to confirm that applications run and meet user requirements.

\section{CONCLUSIONS}

The cloud computing has the significant scope to change the whole education system. In present scenario the e-learning is getting the popularity and this application in cloud computing will surely help in the development of the education offered to poor people which will increase the quality of education offered to them. This paper proposed the design and development of mobile applications in the cloud. The future studies will include issues listed as follows: Enhance the functionality of the system according to user preference, Enhance analysis of the responses from students for learning performance evaluations., Shorten the response time between the front and the backend service toolkit.

\section{ACKNOWLEDGMENTS}

This paper is supported by Humanities Social Sciences Project of Education Department of Hubei Province (No. 14Q092), National Natural Science Foundation of China(No. 61370092),National Science Foundation of Hubei (No. 2013CFC005, No. 2014CFB188), Hubei Provincial Department of Education Outstanding Youth Scientific Innovation Team Support Foundation (No. T201410).

\section{REFERENCES}

[1] Bacigalupo, David; Wills, Gary; De Roure, David; Victor, 2010.A Categorization of Cloud Computing Business Models: IEEE/ACM .

[2] Minutoli, G. Fazio, M. Paone, M. Puliafito, A,2010. Engineering Fac, Univ. of Messina, Messina, Italy Virtual Business Networks With Cloud Computing and Virtual Machines: IEEE/ICUMT . 
[3] Paul Hofmann, SAP Labs, Dan Woods, CITO Research,2010: The Limits of Public Clouds for Business Applications: Digital Library November.

[4] Uhlig,R., Neiger, G. Rodgers, D. S.M. Kagi, A.Leung, F.H. Smith 2005: Intel Corp., USA : Intel visualization technology IEEE Computer Society .

[5] Perez, R., van Doom, L., Sailer, R. IBM T.J,2008. Watson Res. Center, Yorktown Heights, NY : Visualization and Hardware-Based Security.

[6] GregorPetri2010: The Data Center is dead; long live the Virtual Data Center? Join the Efficient Data Center.

[7] The Independent Cloud Computing and Security http://cloudsecurity.org/forum/stats/-Augast 2010.

[8] Dr. Rao Mikkilineni \& Vijay Sarathy, Kawa Objects 2009, Inc. CloudComputing and Lessons: IEEE
[9] T. H. Dihn, C. Lee, D. Niyato, and P. Wang,2011, “A Survey of Mobile Cloud Computing: Architecture, Applications, and Approaches,"Wireless Communications and Mobile Computing - Wiley,DOI:10.1002 WCM/1203, pp. $1-38$.

[10] N. M. Rao, C. Sasidhar, and V. S. Kumar, , 2010 "Cloud ComputingThrough Mobile Learning," (IJACSA) Internationa Journal of Advanced Computer Science and Applications, Vol 1, No. 6, pp. $42-46$.

[11] High Performance Computing, Coraid solutions.

[12] http://www.coraid.com/solutions/high_performance_comp uting[retrieved: 05, 2012]

[13] Maryland CPU-GPU Cluster Infrastructure.

[14] http://www.umiacs.umd.edu/research/GPU/facilities.html[ retrieved: 05, 2012] 